\title{
Perfil de trabalhadores com lombalgia atendidos em um serviço de saúde
}

\section{Profile of workers with lumbar affections attended at a health service}

\author{
José Martim Marques Simas ${ }^{1}$ (1) \\ Maria do Carmo Vitório Alves Ramos ${ }^{2}$ (b) \\ Felipe Granado de Souza 3 (i) \\ Maria do Carmo Baracho de Alencar ${ }^{4}$ (1)
}

\begin{abstract}
${ }^{1}$ Autor para correspondência. Centro Universitário do Vale do Ribeira (Registro). São Paulo, Brasil. simasjmm@gmail.com 2Saúde Integrada Santos (Santos). São Paulo, Brasil. fisio_mariaramos@hotmail.com 3-4Universidade Federal de São Paulo (Santos). São Paulo, Brasil. unifesp.felipe@gmail.com, alencar@unifesp.br
\end{abstract}

\begin{abstract}
RESUMO | INTRODUÇÃO: As transformações no mundo do trabalho têm propiciado o acúmulo de tarefas, exigências de polivalência e aumento do ritmo de trabalho, ocasionando um aumento de lombalgias. OBJETIVO: Descrever o perfil de trabalhadores com lombalgia, atendidos em uma unidade especializada em Saúde do Trabalhador da Baixada Santista. MÉTODOS: Trata-se de um estudo observacional transversal e exploratório. Ocorreu análise documental de prontuários abertos de trabalhadores atendidos no Centro de Referência em Saúde do Trabalhador (CEREST) de Santos-SP, no período de julho de 2014 a julho de 2015, com queixa de lombalgia e diagnósticos nosológicos estabelecidos para "outras dorsopatias" (M51 a M54) segundo a CID-10. Foram obtidos os dados: demográficos, variáveis clínicas, categorias profissionais, entre outras. RESULTADOS: De 502 prontuários, 21,9\% $(n=110)$ apresentaram queixa de lombalgia e diagnóstico clínico pela CID-10. Houve predomínio do sexo feminino (58,2\%), na faixa etária de 35 a 45 anos $(50,9 \%)$ e baixa escolaridade, com ensino fundamental incompleto (35,4\%). Ainda, $23,7 \%$ dos sujeitos estavam em situação de afastamento do trabalho e 85,0\% com registro em carteira de trabalho (CLT). As categorias profissionais mais prevalentes foram de serviços domésticos, 28,3\% $(n=31)$ e atividades de limpeza, 19,1\% ( $n=21)$. Dos sujeitos, 68,2\% tiveram assistência de Fisioterapeutas. CONCLUSÃO: A prevalência foi maior no sexo feminino, de trabalhadores formais e afastados do trabalho, do setor de serviços domésticos e atividades de limpeza, de baixa escolaridade, e com a faixa etária considerada produtiva para o trabalho.
\end{abstract}

PALAVRAS-CHAVE: Doenças da coluna vertebral. Dor lombar. Saúde do trabalhador. Serviço de saúde. Saúde coletiva.

\begin{abstract}
INTRODUCTION: The changes in the world of work haved a significant impact on tasks, capturing versatility and increasing the pace of work, causing an increase in low back pain. OBJECTIVE: To describe the profile of workers with low back pain treated at a unit specialized in Occupational Health in Baixada Santista. METHODS: This is an observational cross-sectional and exploratory study. A documentary analysis of the open records of workers attended at the Reference Center for Occupational Health (CEREST), in Santos-SP, from July 2014 to July 2015 was carried out, with a list of low back pain and diagnosis in the following clinical methods for "Other dorsopathies" (M51 to M54) according to ICD-10. The data were selected: demographic, clinical variables, professional categories, among others. RESULTS: Of the 502 medical records, $21.9 \%(n=110)$ reported complaints of low back pain and clinical diagnosis by ICD-10. There was a predominance of females (58.2\%), aged between 35 and 45 years (50.9\%) and low education, with incomplete primary education (35.4\%). Still, $23.7 \%$ of individuals were absent from work and $85.0 \%$ were registered on the work card (CLT). The most prevalent professional categories were domestic services, $28.3 \%(n=31)$ and cleaning activities, $19.1 \%(n=21)$. Of the subjects, $68.2 \%$ had assistance from physiotherapists. CONCLUSION: The prevalence was higher in women, in workers trained and away from work, in the sector of domestic services and cleaning activities, in low education and in the age group considered productive for work.
\end{abstract}

KEYWORDS: Spinal diseases. Lumbar pain. Worker's health. Health service. Public health. 


\section{Introdução}

A precarização do trabalho vem avançando, com maior exploração do trabalho e o decréscimo de amparo social aos trabalhadores, seguindo as novas lógicas de gestão do capital, e com isso muitos adoecimentos vem surgindo entre os trabalhadores ${ }^{1}$. Isso afeta a qualidade de vida e tem ocasionado distúrbios osteomusculares relacionados ao trabalho (DORT) e lombalgias ocupacionais em trabalhadores de diversas categorias profissionais que desempenham atividades com demandas psicossociais e sobrecarga física de trabalho, como força, repetitividade e posturas inadequadas 2 .

Neste sentido, entre as doenças do trabalho estão as dorsopatias, sendo um problema de saúde pública em vários países e que causam prejuízos econômicos para a sociedade ${ }^{3}$. As dorsopatias são categorizadas majoritariamente em: cervicalgias, dores torácicas, transtornos dos discos intervertebrais, espondiloses, radiculopatias e as lombalgias que são as mais comuns ${ }^{3}$. As doenças da coluna lombar têm representado uma das principais causas de incapacidade e de afastamento das atividades de trabalho, por motivo de doença4. Aproximadamente $60 \%$ a $80 \%$ da população é acometida pelo quadro de lombalgia durante a vida ${ }^{5}$.

As lombalgias são caracterizadas por dores referidas entre as margens costais e a prega glútea, e usualmente acompanhada de limitação dolorosa ao movimento, além de que é considerada um importante problema de saúde pública, tendo em vista que pode interferir nas relações sociais, econômicas, profissionais e culturais ${ }^{6}$. Ainda, é considerada a maior causa de transtornos de saúde relacionados ao trabalho, incapacitando trabalhadores com menos de 45 anos, podendo também ocasionar invalidez prematura ${ }^{7}$.

Vários são os fatores de risco para as lombalgias. Entre as exposições ocupacionais de risco encontram-se a posição sentada por tempo prolongado, o trabalho físico intenso e pesado, exigências de movimentos de rotação e flexão de tronco durante as atividades, ações de empurrar e puxar objetos, além da exposição à vibração de corpo inteiro ${ }^{8}$. Também se encontram os fatores relacionados à organização do trabalho, como ritmo acelerado, realização de movimentos repetitivos, não valorização do trabalho pela chefia e por outros profissionais, e questões conflituosas de relacionamento interpessoal e do trabalho em equipe? Algumas profissões são de risco para as lombalgias, entre elas estão: motoristas de ônibus, mecânicos, operadores de máquinas, e trabalhadores vinculados aos setores de serviços gerais e de limpezaํ․

Cabe salientar que a lombalgia se torna crônica quando os sintomas são recorrentes por mais de 12 semanas, e cerca de 5 a $10 \%$ dos casos de lombalgias agudas tornam-se crônicas ${ }^{10}$. Os casos crônicos de dor lombar geram aumento nos gastos com recursos em saúde, como também ausências e afastamentos do trabalho geradas pelas incapacidades nas funções físicas, mentais e sociais ${ }^{6}$. A dor lombar crônica impede muitas vezes a execução satisfatória de atividades de vida diária, como de trabalho e até do lazer, fazendo com que os sujeitos acometidos se sintam com medo de realizar as suas atividades ${ }^{11}$.

Este estudo teve o objetivo de descrever o perfil de trabalhadores com lombalgia, atendidos em uma unidade especializada em Saúde do Trabalhador da Baixada Santista.

\section{Métodos}

Trata-se de um estudo observacional transversal e exploratório, descritivo e com resultados quantitativos. Foi realizado em um Centro de Referência em Saúde do Trabalhador (CEREST), da cidade de SantosSP. Este serviço compõe a Rede Nacional de Atenção Integral à Saúde do Trabalhador (RENAST) e atende aos trabalhadores com suspeita de doenças que estejam relacionadas ao trabalho ou submetidos a ameaças, seja física ou mental, de qualquer categoria profissional, com ou sem registro em carteira de trabalho, vinculados à serviços públicos ou privados, como também na situação de autônomos ou desempregados. Além disso, o CEREST de Santos é uma unidade regional que foi criada em 1990, com território de abrangência e de ações que agregam os municípios de Santos, São Vicente e Praia Grande.

Ocorreu o levantamento de dados de prontuários abertos de trabalhadores atendidos neste serviço, no período de 01 de julho de 2014 a 01 de julho de 2015, tendo como critérios de seleção: queixa de lombalgia em consulta inicial, de ambos os sexos, e com faixa etária entre 35 a 55 anos. A escolha deste período se deu pelo andamento de um projeto com atuação de fisioterapeutas e realizado junto aos trabalhadores com lombalgia na época. Após a seleção dos prontuários, foram obtidos os seguintes dados: demográficos 
(idade, sexo, escolaridade e município de residência); profissão, queixa em consulta inicial, diagnóstico clínico; e se houve o encaminhamento para o tratamento fisioterapêutico.

Realizou-se o agrupamento dos diagnósticos, conforme a Classificação Internacional de Doenças (CID-10), sendo selecionados para as análises desse estudo, os sujeitos com diagnósticos clínicos referente às "outras dorsopatias" (M50 a M54). Para a categorização profissional foi utilizado o setor/atividade profissional, segundo a Classificação Nacional de Atividades Econômicas (CNAE), que é um instrumento utilizado pelo Instituto Brasileiro de Geografia e Estatística (IBGE), aplicado a todos os agentes econômicos que estão envolvidos na produção de bens e serviços. Os dados foram registrados no programa Microsoft Excel ${ }^{\circledR}$ e depois foi realizada uma análise descritiva dos dados por meio do software R Development Core Team ${ }^{\circledR}$, em que as variáveis categóricas foram apresentadas em tabelas de frequências absolutas e relativas.

O estudo foi devidamente conduzido sob preceitos éticos, com aprovação do Comitê de Ética em Pesquisa da Universidade Federal de São Paulo (UNIFESP), sob parecer número 44711715.8.0000.5505.

\section{Resultados}

Foram analisados 502 prontuários abertos de trabalhadores que foram atendidos no CEREST de Santos, no período de 01 julho de 2014 a 01 de julho de 2015. Destes prontuários, 21,9\% (n=110) trabalhadores apresentavam queixa de lombalgia em consulta inicial e o diagnóstico clínico estabelecido pela CID-10 entre M51 a M54. Na tabela 1 estão demonstrados alguns dados sociodemográficos e situação de emprego registrados.

Tabela 1. Dados sociodemográficos e situação de emprego dos trabalhadores

\begin{tabular}{|c|c|c|}
\hline Variáveis & $\begin{array}{c}N \\
(110)\end{array}$ & $\%$ \\
\hline \multicolumn{3}{|l|}{ Sexo } \\
\hline Feminino & 64 & 58,2 \\
\hline Masculino & 46 & 41,8 \\
\hline \multicolumn{3}{|l|}{ Faixa etária } \\
\hline 35 a 45 anos & 56 & 50,9 \\
\hline 46 a 55 anos & 54 & 49,1 \\
\hline \multicolumn{3}{|l|}{ Escolaridade } \\
\hline Ensino Fundamental Incompleto & 39 & 35,4 \\
\hline Ensino Fundamental Completo & 13 & 19,1 \\
\hline Ensino Médio Incompleto & 10 & 11,1 \\
\hline Ensino Médio Completo & 21 & 23,3 \\
\hline Ensino Superior & 7 & 6,4 \\
\hline Sem Informação no Prontuário* & 20 & 18,1 \\
\hline \multicolumn{3}{|l|}{ Situação de emprego } \\
\hline Afastado do Trabalho (INSS) & 19 & 17,3 \\
\hline Ativo & 10 & 9,1 \\
\hline Em Licença Médica & 7 & 6,4 \\
\hline Desempregado & 9 & 8,2 \\
\hline Sem Informação no Prontuário* & 65 & 59 \\
\hline
\end{tabular}


De acordo com os dados demonstrados na tabela 1, a maioria $(58,2 \%)$ dos trabalhadores eram do sexo feminino. Alguns sujeitos se encontravam em situação de afastamento do trabalho pelo Instituto Nacional do Seguro Social (INSS) $(17,3 \%)$, alguns em licença médica $(6,4 \%)$, e outros estavam ativos no trabalho (9,1\%). Além disso, $85,0 \%$ dos sujeitos tinham registro em carteira de trabalho (CLT). Além disso, a maioria dos trabalhadores residiam na cidade de Santos-SP (96,3\%).

Na tabela 2 estão demonstrados os diagnósticos clínicos encontrados junto aos sujeitos.

Tabela 2. Diagnósticos clínicos dos trabalhadores encontrados e estabelecidos entre M50 a M54 (CID-10)

\begin{tabular}{|c|c|c|c|}
\hline & $\begin{array}{l}\text { Diagnósticos Clínicos } \\
\text { (Código CID-10) }\end{array}$ & $\begin{array}{c}N \\
(110)\end{array}$ & $\%$ \\
\hline M54.5 & Dor Lombar Baixa & 60 & 54,5 \\
\hline M51.2 & Outros Deslocamentos Discais Intervertebrais Especificados & 37 & 33,6 \\
\hline M54.4 & Lumbago com Ciática & 20 & 18,2 \\
\hline M54.6 & Dor na Coluna Torácica & 14 & 12,7 \\
\hline M51 & Outros Transtornos de Discos Intervertebrais & 11 & 10,0 \\
\hline M54.2 & Cervicalgia & 8 & 7,3 \\
\hline M54 & Dorsalgia & 4 & 3,6 \\
\hline M50.8 & Outros Transtornos de Discos Cervicais & 4 & 3,6 \\
\hline M53.1 & Síndrome Cervicobraquial & 4 & 3,6 \\
\hline M51.1 & Transtornos de Discos Lombares e de Outros Discos Intervertebrais com Radiculopatias & 3 & 2,7 \\
\hline M50.3 & Outra Degeneração de Disco Cervical & 1 & 0,9 \\
\hline M51.4 & Nódulos de Schmorl & 1 & 0,9 \\
\hline M54.1 & Radiculopatia & 1 & 0,9 \\
\hline M54.8 & Outra Dorsalgia & 1 & 0,9 \\
\hline M54.9 & Dorsalgia Não Especificada & 1 & 0,9 \\
\hline
\end{tabular}

Os diagnósticos clínicos mais prevalentes entre os trabalhadores foram M54.5 (dor lombar baixa) com 54,5\%, M51.2 (outros deslocamentos discais intervertebrais especificados) com 33,6\%, e M54.4 (lumbago com ciática) com 18,2\%. Além disso, 39,1\% apresentaram mais de um diagnóstico clínico relacionado às "outras dorsopatias".

Na tabela 3 encontram-se os dados referentes às atividades profissionais, de acordo com a Classificação Nacional de Atividades Econômicas (CNAE).

Tabela 3. Categorias profissionais dos trabalhadores de acordo com a CNAE

\begin{tabular}{|c|c|c|}
\hline Setor/Atividade Profissional & $\begin{array}{c}N \\
(110)\end{array}$ & $\%$ \\
\hline Serviços domésticos & 31 & 28,3 \\
\hline Atividades de limpeza & 21 & 19,1 \\
\hline Outros setores & 15 & 13,6 \\
\hline Fornecimento e gestão de recursos humanos para terceiros & 11 & 10,0 \\
\hline Publicidade & 5 & 4,5 \\
\hline Armazenamento, carga e descarga & 4 & 3,6 \\
\hline Atividades de vigilância, segurança privada e transporte de valores & 3 & 2,7 \\
\hline Outros serviços especializados para construção & 3 & 2,7 \\
\hline Sem informação no prontuário & 17 & 15,5 \\
\hline
\end{tabular}


O setor/atividade de trabalho mais encontrado foi o de "serviços domésticos", com 28,3\%, seguido de "atividades de limpeza", com 19,1\%, e "outras" com 13,6\%. Diversas profissões foram encontradas nos prontuários, entre elas: auxiliar de limpeza (10,0\%), empregada doméstica (10,0\%), faxineira $(7,2 \%)$, ensacador $(4,5 \%)$. Entre as outras atividades estiveram: ajudante geral, auxiliar de serviços gerais, caminhoneiro, cozinheira, cuidador (a) de idosos, vigilante com percentuais variando entre 0,9 a 1,8\%. Ademais, cabe ressaltar que em somente $8,1 \%$ dos prontuários havia o registro de comunicado de acidente de trabalho (CAT).

Além disso, 68,2\% receberam tratamento fisioterapêutico, e os demais, 31,8\% apenas tiveram a indicação de tratamento medicamentoso. E dos sujeitos que foram encaminhados para algum fisioterapeuta, a maioria era do sexo feminino $(58,7 \%)$.

\section{Discussão}

Muitos trabalhadores apresentaram a queixa de lombalgia em consulta inicial, porém sem o registro conclusivo de um diagnóstico clínico, por este motivo foram selecionados somente 110 prontuários. Seria importante para o serviço e usuário obter um diagnóstico clínico para o tratamento, como também um diagnóstico funcional e fisioterapêutico, porém nem sempre os sujeitos retornavam ao serviço com os exames clínicos solicitados, talvez por se "perderem" na rede de serviços, indicando dificuldades na intersetorialidade entre os serviços de saúde, havendo a necessidade de maiores investigações a este respeito. Em outro estudo realizado no mesmo serviço, a lombalgia também foi prevalente entre os trabalhadores e $8,8 \%$ dos prontuários analisados $(\mathrm{n}=431)$ também não constavam um diagnóstico clínico estabelecido ${ }^{12}$.

Em relação ao sexo, a maioria dos trabalhadores eram do sexo feminino. Há uma maior prevalência de dor lombar no sexo feminino, e tendendo à maior progressão, quanto maior for a idade ${ }^{13}$. A alta prevalência de dor lombar no sexo feminino pode ocorrer pelo fato das mulheres estarem expostas principalmente à repetitividade, posição viciosa, e trabalho com exigências de grande velocidade em movimentos e repetitivos $6,8,9,14,15$. Várias categorias profissionais já foram apontadas com maiores prevalências para as lombalgias, sendo muitas delas desempenhadas majoritariamente por mulheres, como serviços dos setores domésticos, limpeza, alimentação, informal (diaristas) e têxtil (costureiras)².

Além disso, a maioria dos trabalhadores tinham idade acima de 40 anos. As lombalgias crônicas são mais comuns nas faixas etárias acima de 40 anos $s_{1,8,10}$, e há relações entre o aumento de idade e a maior frequência de incapacidade por dor lombar, devido ao fato do envelhecimento tender a provocar mudanças degenerativas e progressivas na coluna vertebral 6,8 . Em relação ao sexo feminino, as mulheres estariam mais expostas às maiores cargas ergonômicas e com trabalhos repetitivos ${ }^{3}$, justificando entre outros fatores a incidência elevada de dor lombar. Outro fator de risco está relacionado à baixa escolaridade e às profissões menos especializadas e que não exigem muitas qualificações ${ }^{\underline{6}}$.

Dos prontuários que continham a informação do nível de escolaridade $(81,9 \%)$, a maioria $(65,6 \%)$ apresentou baixa escolaridade, considerada até o ensino médio incompleto. Obstante a isso, a baixa escolaridade é um dos fatores de risco para lombalgias por predispor os trabalhadores às exigências no trabalho com esforços físicos e repetitivos ${ }^{16}$. A escolaridade tem sido considerada um importante fator de risco para a lombalgia, sendo inversamente correlacionadas, ou seja, quanto menor à escolaridade, maior a tendência à lombalgia e quanto maior a escolaridade, menor a probabilidade ${ }^{8}$. Hartvigsen et al. (2018) ${ }^{8}$ sugerem que os mecanismos que explicam os efeitos da baixa escolaridade na lombalgia incluem exposições ambientais e de estilo de vida em grupos socioeconômicos diferentes, menor conhecimento e educação em saúde, que não estão disponíveis ou adequadamente direcionados às pessoas com baixa escolaridade. Eles reforçam ainda que, na rotina das pessoas com baixa escolaridade, tanto no domicílio, como no trabalho, é comum a presença de ocupações variadas e com aumento da carga física de trabalho, que também são fatores de risco para a dor lombar. 
Em relação à situação de trabalho, e dos prontuários que continham este registro, $17,3 \%$ se apresentavam em situação de afastamento do trabalho, que pode estar atrelado à cronicidade da doença, que pode dificultar o reingresso ao mercado de trabalho ${ }^{17}$. Esta situação de afastamento do trabalho pode gerar sofrimento aos trabalhadores, pela perda do papel social, pelas dificuldades em afazeres domésticos, antes facilmente realizáveis, e pelos possíveis conflitos familiares decorrentes das limitações físicas impostas pela doença ${ }^{11}$.

A dor nas costas idiopática foi a primeira causa de invalidez e de auxílio-doença no Brasil, e o diagnóstico de Dorsalgia (M.54) apresentou elevada incidência, pela não especificação do local de dor ${ }^{16}$. Entende-se dessa forma que a ausência de diagnóstico clínico expresso no prontuário pode ser um fator de dificuldade para aprofundamentos investigativos e clínicos para os fisioterapeutas, cabendo reflexões visando melhorias nos registros dos prontuários. Em relação aos diagnósticos clínicos apontados, os mais prevalentes foram: M54.4 com 54,5\%, M51.2 com 33,6\%, e M54.4 com 18,2\%. O diagnóstico de dor lombar baixa (M54.5) está dentro da subclasse das dorsalgias (M54). E a dor lombar baixa está entre as doenças relacionadas ao trabalho ${ }^{18}$.

Em relação aos setores/atividades registrados, quase a metade dos trabalhadores $(47,4 \%)$ estavam vinculados ao setor de serviços domésticos e de atividades de limpeza. Em alguns estudos, já foram apontadas maiores prevalências de dores lombares em trabalhadores que executam a atividade de limpeza ${ }^{11,16,19,20}$. Diversas ocupações, muitas vinculadas às más condições de trabalho, estão relacionadas com a presença de lombalgias. Com as mudanças que tem ocorrido no mundo do trabalho, houve nos diversos setores da sociedade, com insurgência da informalidade, flexibilizações, multitarefas e precarização das condições de trabalho, pautadas em políticas neoliberais, alicerçadas nas falas do desenvolvimento econômico global'. Com isso, quadros de lombalgias e incapacitações funcionais tornam-se objeto de grande preocupação em políticas públicas, especialmente em países pobres ou em desenvolvimento, como o Brasil. A incapacidade por dor lombar é mais alta nas faixas etárias produtivas para o trabalho em todo o mundo, o que é especialmente preocupante nos países de baixa e média renda em que o emprego informal é comum e as possibilidades de modificação no emprego são limitadas .
Além disso, foi notado um percentual baixo $(8,1 \%)$ de notificação para os acidentes de trabalho, o que possivelmente pode estar atrelado à subnotificação de CAT. Figueira et al. (2017) ${ }^{20}$ expressam que as notificações de acidentes de trabalho não refletem a realidade, havendo uma estimativa de que para cada caso notificado, nove não foram notificados. Eles reforçam que situações relacionadas com distúrbios musculoesqueléticos tendem a ser ocultadas, já que não é considerado simples a relação de nexo causal com o trabalho. Ainda, apesar de haver outras formas para a emissão da CAT, esta situação comumente fica sob a decisão do empregador, sendo importante também a análise do comportamento dos empregadores frente às comunicações e também acerca de ações preventivas, cabendo maiores fiscalizações, investigações e estudos.

No contexto sobre os encaminhamentos para tratamento fisioterapêutico, 68,2\% dos trabalhadores foram encaminhados. Os tratamentos para dores lombares realizados por fisioterapeutas são muito importantes, sendo fundamentais em casos crônicos, pois visam uma melhor qualidade de vida aos pacientes ${ }^{21-23}$. Além disso, a maioria dos trabalhadores encaminhados para este serviço era do sexo feminino. O maior percentual de procura pelo tratamento fisioterapêutico é de mulheres, quando comparadas aos homens, aspecto que indica um padrão cultural, onde a mulher se mostra mais preocupada com o cuidado que o homem²2, situação que merece maiores aprofundamentos em estudos futuros.

O estudo apresentou limitações, por se tratar de um único serviço de saúde e de seleção de um agrupamento de diagnósticos clínicos e em um determinado período. Uma dificuldade encontrada, foi a falta de informação de alguns dados nos prontuários (dados de escolaridade e situação atual de emprego), sendo o serviço notificado na época, visando aprimoramentos. Porém ressalta-se a importância dessas informações para futuros aprofundamentos, como também uma maior atuação e engajamento dos fisioterapeutas, com análise e perspectiva para avaliação da saúde funcional dos sujeitos, podendo-se inclusive utilizar a classificação internacional de incapacidade e funcionalidade em saúde (CIF). 


\section{Conclusão}

O estudo possibilitou descrever os dados sobre o perfil de trabalhadores acometidos com lombalgia e atendidos em um serviço especializado em saúde do trabalhador na Baixada Santista. A prevalência foi maior no sexo feminino, de trabalhadores formais e afastados do trabalho, do setor de serviços domésticos e atividades de limpeza, de baixa escolaridade, e com a faixa etária considerada produtiva para o trabalho. Futuras pesquisas podem aprofundar as informações trazidas junto aos trabalhadores com lombalgia, e em outros serviços de saúde.

\section{Contribuições dos autores}

Simas JMM participou da análise dos dados da pesquisa, interpretação dos resultados e redação do artigo científico. Ramos MCVA participou da coleta de dados da pesquisa e interpretação dos dados. Souza FG participou da análise estatística dos dados da pesquisa e interpretação dos resultados. Alencar MCB participou da concepção, delineamento, análise e interpretação dos dados da pesquisa, e redação do artigo científico.

\section{Conflitos de interesses}

Nenhum conflito financeiro, legal ou político envolvendo terceiros (governo, empresas e fundações privadas etc.) foi declarado para nenhum aspecto do trabalho submetido (incluindo, mas não se limitando a subvenções e financiamentos, participação em conselho consultivo, desenho de estudo, preparação de manuscrito, análise estatística etc.).

\section{Referências}

1. Antunes R, Praun L. A sociedade dos adoecimentos no trabalho. Serv Soc Soc. 2015;(123):407-27. doi: 10.1590/0101-6628.030

2. Paula EA, Buschinelli JT, Maeno M, Costa RF. Qualidade de vida de trabalhadores com LER/DORT e lombalgia ocupacional atendidos no Cerest de Guarulhos, São Paulo. Rev Br Saúde Ocup. 2016;41:(e19):1-11. doi: 10.1590/2317-6369000120115

3. Ferreira GD, Silva MC, Rombaldi AJ, Wrege ED, Siqueira FV, Hallal PC. Prevalência de dor nas costas e fatores associados em adultos do Sul do Brasil: estudo de base populacional. Rev Bras Fisioter. 2011;15(1):31-6. doi: 10.1590/S1413-35552011005000001
4. Finger ME, Selb M, Bie RD, Escorpizo R. Using the International Classification of Functioning, Disability and Health in Psysiotherapy in Multidisciplinary Vocational Rehabilitation: a case study of low back pain. Psyother Res Int. 2015;20(4):231-41. doi: 10.1002/pri.1587

5. Zavarize SF, Wechsler SM, Lima AB, Martelli A. Dor lombar crônica: implicações do perfil criativo como estratégia de enfrentamento. JMPHC. 2014;5(2):188-194. doi: 10.14295/jmphc. $\underline{\mathrm{v} 5 \mathrm{i} 2.215}$

6. Bento TPF, Genebra CVS, MMaciel NM, Cornelio GP, Simeão SFAP, Vitta A. Low back pain and some associated factors: is thereany difference between genders? $\mathrm{Br} \mathrm{J}$ Phys Ther. 2020;24(1):79-87. doi: 10.1016/j.bjpt.2019.01.012

7. Helfenstein Junior M, Goldenfum MA, Siena C. Lombalgia ocupacional. Rev Ass Med Bras. 2010;56(5):583-9.

8. Hartvigsen J, Hancock MJ, Kongsted A, Louw Q, Ferreira ML, Genevay $S$ et al. What low back pain is and why we need to pay attention. The Lancet. 2018; (10137):2356-2367. doi: 10.1016/ S0140-6736(18)30480-X

9. Martins JT, Ribeiro RP, Bobroff MCC, Marziale MHP, Robazzi MLCC, Mendes AC. Significados das cargas de trabalho sob a ótica de profissionais de limpeza. Acta Paul Enferm. 2013;26(1):63-70. doi: 10.1590/S0103-21002013000100011

10. Momsen AMH, Jensen OK, Nielsen CV, Jensen C. Multiple somatic symptoms in employees participating in a randomized controlled trial associated with sickness absence due to nonspecific low back pain. Spine J. 2014;14(12): 2868-76. doi: 10.1016/j.spinee.2014.01.062

11. Valença JBM, Alencar MCB. O afastamento do trabalho por dor lombar e as repercussões na saúde: velhas questões e desafios que continuam. Cad Bras Ter Ocup. 2018;26(1):119-127. doi: 10.4322/2526-8910.ctoA01074

12. Alencar MCB, Valença JBM, Ferraz KP, Souza FG, Lopes LV. Perfil de trabalhadores com doenças da coluna vertebral atendidos em um serviço de saúde. Cad Ter Ocup UFSCar. 2016;24(2):227-233. doi: 10.4322/0104-4931.ctoAO0575

13. Leopoldino AAO, Diz JBM, Martinsb VT, Henschke N, Pereira LSM, Dias RC et al. Prevalence of low back pain in older Brazilians: a systematic review with meta-analysis. Rev Bras Reum. 2016;56(3):258-69. doi: 10.1016/j.rbre.2016.03.011

14. Sowah D, Boyko R, Antle D, Miller L, Zakhary M, Straube S. Occupational interventions for the prevention of back pain: Overview of systematic reviews. J Safety Res. 2018;(66):39-59. doi: 10.1016/j.jsr.2018.05.007 
15. Silva T, Mills K, Brown BT, Pocovi N, Campos T, Maher C et al. Recurrence of low back pain is common: a prospective inception cohort study. J Phys. 2019;65(3):159-165. doi: 10.1016/j. jphys.2019.04.010

16. Anderson SP, Oakman J. Allied health professionals and workrelated musculoskeletal disorders: a systematic review. Saf Health Work. 2016;7(4):259-67. doi: 10.1016/j.shaw.2016.04.001

17. Campbell P, Wynne-Jones G, Muller S, Dunn KM. The influence of employment so $\neg$ cial support for risk and prognosis in nonspecific back pain: review and critical synthesis. Int Arch Occup Envir Health. 2013;86(2):119-37. doi: 10.1007/s00420-0120804-2

18. Meziat Filho N, Silva GA. Inva-lidez por dor nas costas entre segurados da Previdência Social do Brasil. Rev Saúde Publica. 2011;45(3):494-502. doi: 10.1590/S0034-89102011000300007

19. Pataro SMS, Fernandes RCP. Trabalho físico pesado e dor lombar: a realidade na limpeza urbana. Rev Bras Epidemiol. 2014;17(1):17-31. doi: 10.1590/1809-4503201400010003ENG

20. Figueira VA, organizador. Saúde e segurança do trabalho no Brasil. Brasília: Movimento; 2017. P. 476.

21. Silva GG, Sirena SA. Perfil de encaminhamentos à fisioterapia por um serviço de atenção primária à saúde. Epidemiol Serv Saúde. 2015;24(1):123-33. doi: 10.5123/S1679. 49742015000100014

22. Sacon AB, Pillatt AP, Berbam LW, Fengler VZ, Bigolin SE. Perfil de sujeitos atendidos na clínica-escola de fisioterapia na área de ortopedia e traumatologia. Revista Contexto \& Saúde. 2011;10(20):1191-6. doi: 10.21527/2176-7114.2011.20.1191-1196

23. Santos JKV, Gomes Junior VFF, Souza AS, Farias NS, Marques SS, Costa JM. Socio-demographic and physical-functional profile of low back pain patients assisted in Manaus-AM. Rev Dor.

2015;16(4):272-5. doi: 10.5935/1806-0013.20150055 